\title{
PREDISPOSICIÓN HACIA LAS EXPERIENCIAS ALUCINATORIAS EN PERÚ: EXAMINANDO LA CONTINUIDAD NORMALIDAD- PATOLOGÍA EN INDIVIDUOS CLÍNICOS Y NO CLÍNICOS
}

\author{
Alejandro Parra ${ }^{1}$, Luis Espinoza P. \\ ${ }^{1}$ Instituto de Psicología Paranormal, Buenos Aires, Argentina y \\ Núcleo de InVestigación en Psicología Anómala, Lima, Perú \\ (RECBiIDO EL 26/5/2009, ACEPTADO EL 9/11/ 2009)
}

\begin{abstract}
RESUMEN
Recientemente se ha observado un creciente interés en el estudio de las alucinaciones en población no-clínica. En este estudio, se investigó la multidimensionalidad de las alucinaciones en participantes no-clínicos, en comparación con participantes clínicos. Ambas muestras -214 estudiantes universitarios y 30 pacientes- tomaron parte en este estudio. Se empleó el Cuestionario de Experiencias Alucinatorias. Los resultados sugieren que los estudiantes con experiencias perceptuales anómalas indicaron algún grado de propensión a la esquizotipia perceptual, tendencia a la disociación, absorción y propensidad a la fantasía en relación con la alucinación visual, lo cual parece confirmar la esquizotipia positiva.
\end{abstract}

Palabras clave: Alucinaciones, esquizotipia perceptual, tendencia a la disociación, absorción, propensidad a la fantasía.

\section{ABSTRACT \\ PREDISPOSITION TOWARDS HALLUCINATORY EXPERIENCES IN PERU: EXAMINING THE CONTINUITY-PATHOLOGY IN NORMAL INDIVIDUALS CLINICAL AND NONCLINICAL}

The purpose of the present study is to test whether hallucinatory experiences respond to the dimensionality principle and to detect potential differences between a group of "hallucinators" and a group of "non-hallucinators" with regard to personality traits in 214 Peruvian undergraduates students (non-clinical sample) and 30 outpatients (clinical sample). Among a sample of undergraduates, it was predicted that those who reported a number of anomalous perceptual experiences would score higher than nonexperiencers on the hallucination prone (auditive, visual, hipnagogic/hipnopompic and tactile), fantasy proneness, absorption, dissociation, and the cognitive-perceptual factor of the schizotypal questionnaire. All the predictions were significantly confirmed except those concerning interpersonal and disorganized schizotypy.

Keywords: Hallucinatory experiences, cognitive-perceptual schizotypy, dissociation, absorption, fantasy proneness.

1 Invesitgador del Instituto de Psicología Paranormal. 


\section{INTRODUCCIÓN}

Las alucinaciones son percepciones que ocurren en ausencia de los estímulos sensoriales correspondientes. Algo es percibido, pero objetivamente no hay nada para percibir. Hay tres características que distinguen la alucinación de cualquier otro fenómeno psicológico; en primer lugar, existe la convicción de que el fenómeno tiene su origen fuera de uno mismo, esto es, que se produce en el mundo real. Segundo, hay una falta de control por parte del individuo que intenta distinguir entre las alucinaciones y otras clases de imágenes mentales vívidas (por ejemplo, la imaginación). Finalmente, en las alucinaciones existe una imposibilidad, o por lo menos una dificultad, de alterar o disminuir la experiencia por deseo expreso de la persona. Estas experiencias han sido estudiadas por la psicopatología y suelen ser vistas como experiencias perceptuales patológicas, sin embargo, no hay razón a priori ni justificación teórica alguna para limitar la definición de alucinación al campo de la psicopatología, si bien es más fácil definir fenomenológicamente una alucinación dentro de un contexto psicopatológico, que dentro de un contexto sociocultural.

El primer estudio sistemático por determinar si las alucinaciones podían ocurrir en personas sin enfermedades físicas o mentales fue llevado a cabo por la Society for Psychical Research en Gran Bretaña a fines del siglo XIX (Sidgwick, 1894). Un gran equipo de colaboradores entrevistó a un total de 7717 hombres y 7599 mujeres. Aunque no se hizo ningún muestreo realmente aleatorio, nadie fue excluido de este estudio, tenga o no señales obvias de enfermedad física o mental. Del total, el 7,8\% de la muestra de hombres y el $12 \%$ de mujeres indicaron tener al menos una experiencia alucinatoria vívida; la más común era la modalidad visual: una persona viva que no estaba presente en el momento de la experiencia. También se registraron alucinaciones con contenido religioso o sobrenatural, así como el hecho de que las alucinaciones auditivas no eran tan frecuentes como las visuales.

En otro estudio, Posey y Losch (1983) entrevistaron a 375 estudiantes universitarios y encontraron que el $39 \%$ mostraba el síntoma schneideriano de primer rango (haber oído a alguien que hablaba en voz alta), y el 5\% tener conversaciones con sus alucinaciones. Encuestas de estudiantes posteriores a ésta, llevadas a cabo en Gran Bretaña (Bentall y Slade, 1985a, 1985b; Young, Bentall, Slade y Dewey, 1986) y en Estados Unidos (Barret y Etheridge, 1992, 1994), también revelaron que una sorprendente proporción de individuos tenía experiencias alucinatorias. Los casos de alucinaciones de Barrett y Etheridge (1992, 1994) mostraron niveles elevados de efectos negativos.

Tien (1991) condujo la más esclarecedora encuesta de alucinaciones en población general hasta hoy. Esta encuesta estuvo a cargo del Centro de Investigaciones Epidemiológicas (CIE), basado en un cuestionario de síntomas psiquiátricos sobre una población seleccionada aleatoriamente. Se entrevistó a un total de 18572 personas, y un año más tarde se entrevistaron nuevamente a 15 258. En este estudio la definición de alucinación estaba basada en la tercera edición del DSM, que difiere poco de la del DSM-IV. Tien comparó los datos del CIE con los de Sidgwick (1894). Los resultados de ambos estudios mostraron ser muy similares. Tien estima que la prevalencia de alucinaciones en el estudio del CIE fue de $13 \%$ en la primera evaluación y $11.1 \%$ en la segunda. Como en el estudio de Sidgwick, la alucinación en las mujeres era el doble que en los hombres. La principal diferencia entre ambos estudios tiene que ver tanto con el rango etario de los sujetos alucinadores, 
más frecuente en la tercera edad, como en la prevalencia de alucinaciones de tipo visual, menos frecuente en el estudio de Sidgwick.

Por supuesto, quienes sostienen que la esquizofrenia representa el extremo de un continuo de características de personalidad consideran que las personas "normales" que tienen alucinaciones deben ser clasificadas como esquizoides. Sin embargo, el hecho de que una minoría de la población haya tenido experiencias alucinatorias en algún momento de sus vidas debe considerarse como algo digno de atención. Por lo tanto, por cada persona que recibe un diagnóstico de esquizofrenia, casi diez han tenido alucinaciones sin recibir diagnóstico (Aleman \& Haan, 1998).

De manera similar al estudio anterior, Leudar, Thomas, McNally y Glinsky (1997) examinaron a pacientes esquizofrénicos con alucinaciones no-psiquiátricas y sorprendentemente encontraron pocas diferencias entre muestras de pacientes y no-pacientes. En ambos grupos, la mayoría indicó que sus voces intentaban controlar sus actividades cotidianas, por ejemplo, diciéndoles qué hacer o qué no hacer, o dándoles instrucciones para determinados actos; rara vez las voces eran extrañas y por lo general pertenecían a personas que habían sido significativas para sus vidas. Estos estudios sugieren que puede no ser la naturaleza de las experiencias alucinatorias per se lo que determina si una persona va a convertirse en paciente psiquiátrico o no, sino la forma en que las personas reaccionan a sus experiencias.

Hoy día, las definiciones de alucinación han cambiado, por ejemplo, la cuarta edición del DSM (American Psychiatric Association, 1994) define alucinación como "una percepción sensorial que tiene el sentido real de una percepción verdadera pero que ocurre sin la estimulación externa de un órgano sensorial relevante”. Más recientemente, Bentall (2000) propone una definición más precisa como: "Cualquier experiencia parecida a una percepción, la cual (a) ocurra en ausencia de un estímulo apropiado, (b) tenga la fuerza completa o el impacto de su correspondiente (o real) percepción, y (c) no sea permeable al control voluntario de quien la experimenta." Esta definición tiene la ventaja de reconocer el papel de la estimulación ambiental que puede tener lugar en la experiencia de la alucinación.

Algunos autores han intentado distinguir entre "verdaderas" alucinaciones y "pseudoalucinaciones", definidas así porque son percibidas cuando ocurren desde dentro del cuerpo o porque se reconoce que no son verídicas para el percipiente. El DSM-IV distingue entre el hecho de que una persona con alucinaciones auditivas pueda reconocer si una persona está teniendo una experiencia sensorial falsa y donde está convencida de que la fuente del estímulo es independiente de la realidad física. Algunos clínicos e investigadores incluyen [como alucinaciones] las que provienen desde dentro de la cabeza. Sin embargo, el DSM sostiene que ninguna de estas distinciones tiene utilidad clínica (Bentall, 2000; Watkins, 2003).

La experiencia alucinatoria no sólo parece estar ampliamente distribuida en la población en general, sino que también algunos estudios han demostrado una relación cercana entre la incidencia de estas experiencias y sus correlatos psicológicos y de personalidad. La mayor parte de estos estudios muestran que los individuos saludables que tienen tales experiencias tienen mayor nivel de esquizotipia cognitivo-perceptual, absorción psicológica, disociación, propensidad a la fantasía, e imaginería sin alcanzar naturalmente los niveles críticos que se encuentran en la psicosis. 
Por ejemplo, Tellegen (1982) ha observado que las personas que tienen alta capacidad de absorción también tienen imaginería intensa y vívida, y tienen experiencias sensoriales e imaginativas tan intensas al punto de perder su sentido del yo (por ej. ver una película involucrándose tanto en ésta, que se pierde el contexto que lo rodea, incluso si otro le habla), y definió a la absorción como un rasgo de la personalidad que permite a la persona implicarse en una variedad de experiencias a través de la imaginación.

Estrechamente vinculado al concepto de absorción y sugestionabilidad, la propensidad a la fantasía se define como un tipo de personalidad caracterizado por una profunda implicación en un mundo privado de fantasía, ensoñamiento vívido, recuerdos, experiencias sensoriales y experiencias paranormales (Wilson y Barber, 1981, 1983). Un aspecto de las personas propensas a la fantasía era su habilidad para tener alucinaciones vívidas que se experimentan "tan reales como la propia realidad". Estas experiencias alucinatorias pueden ocurrir involuntariamente, por ejemplo, una mujer entrevistada por Wilson y Barber dijo que mientras conducía, no podía permitirse pensar que un niño se lanzaba a su coche, ya que, por el simple hecho de pensar en ello, podía provocarle dicha alucinación.

La esquizotipia se encuentra íntimamente relacionada con las psicosis, de hecho el término esquizotipia, también conocida como propensidad a la psicosis (Chapman, Edell, y Chapman, 1980), puede ser considerada como una dimensión normal de la personalidad o como un indicador de la predisposición a la psicosis (Claridge, 1997a, 1997b). Varios estudios indican que las experiencias psicóticas se encuentran presentes en población normal, sugiriendo la existencia de un continuo dimensional (Johns y van Os, 2001) entre la población normal y dichas experiencias.

La esquizotipia se enmarca dentro de este modelo (Claridge, 1997b) abarcando desde la personalidad no patológica (salud) hasta la psicosis (enfermedad). Las variaciones a lo largo de este continuo describen diferentes grados de predisposición a los trastornos psicóticos. Dicha vulnerabilidad o predisposición a la esquizofrenia se expresa, por lo tanto, a lo largo de un continuo psicopatológico. La evaluación de la personalidad esquizotípica se encuentra, básicamente, dentro de las investigaciones que permiten detectar, mediante pruebas psicométricas, a aquellos sujetos con probabilidad de desarrollar trastornos del espectro esquizofrénico (la esquizofrenia misma, los trastornos esquizoafectivos o las personalidades esquizoide, paranoide y esquizotípica). Puntuaciones elevadas de esquizotipia parecen indicar cierta propensidad a desarrollar trastornos esquizofrénicos (Chapman, Chapman, Kwapil, Eckblad, y Zinser, 1994; Kwapil, Miller, Zinser, Chapman, y Chapman, 1997).

Se han observado al menos dos dimensiones de esquizotipia: La dimensión positiva, conocida como experiencias perceptuales no convencionales, se refiere a un funcionamiento excesivo o distorsionado de un proceso normal, que incluye varias formas de alucinaciones, ideación paranoide, ideas de referencia y trastornos del pensamiento. En segundo lugar, la dimensión negativa, conocida como anhedonia o déficit interpersonal, se refiere a la disminución o déficit en la conducta normal del individuo que tiene dificultades para experimentar placer a nivel físico y social, aplanamiento afectivo, ausencia de confidentes íntimos y dificultades en sus relaciones interpersonales. 
Se examinará la experiencia alucinatoria de un grupo de pacientes clínicos y un grupo de estudiantes universitarios peruanos. El objetivo de este estudio es examinar las diferencias cuantitativas de la expresión de la alucinación como un continuo de su ocurrencia.

Ponemos a prueba cinco hipótesis específicas: que personas que puntúan alto en experiencias alucinatorias (auditiva, visual, táctil e hipnagógico/hipnopómpica) tienen puntajes altos de (1) propensidad a la esquizotipia (cognitivo-perceptual, interpersonal, y desorganizada), y

(3) tendencia a la disociación, (4) absorción, (5) propensidad a la fantasía en comparación con quienes no las tienen. También esperamos encontrar diferencias significativas entre la población clínica y no clínica en experiencias alucinatorias auditiva, visual, táctil e hipnagógico/hipnopómpica, propensidad a la esquizotipia (cognitivo-perceptual, interpersonal, y desorganizada), tendencia a la disociación, absorción, propensidad a la fantasía en individuos clínicos en mayor nivel que en estudiantes.

\section{MÉTODO}

\section{Participantes}

Muestra no-clínica. Un total de 214 estudiantes de psicología de ambos sexos de la Facultad de Psicología de la Universidad Ricardo Palma, 159 (74\%) mujeres y 55 (26\%) varones, con un rango etario de 17 y 47 años (Media $=20.87 ; \mathrm{SD}=3.51$ ) residentes en Lima y alrededores.

Muestra clínica. Un total de 30 pacientes (extra-murales, no residentes) del Hospital Santa Rosa de Lima, del departamento de Medicina y Psiquiatría en Lima, Perú, 15 (50\%) mujeres y $15(50 \%)$ varones, con un rango etario de 17 y 61 años (Media $=35,63$; SD $=10,75)$ de Lima y alrededores.

\section{Instrumentos}

Ambas muestras completaron cinco escalas relacionadas con experiencias perceptuales inusuales. El Cuestionario de Alucinaciones (CEA) (Parra, Adróver y González, 2006; alfa de Cronbach $=.93$ ) mide la propensidad a alucinar en seis modalidades sensoriales de 38 ítems con una escala Likert 0-5, siendo 0 nunca a 5 frecuentemente (sólo cuatro se usaron: auditivo, por ej, "Cuando estoy completamente solo en casa, oigo una voz que me llama por mi nombre, una sola vez"; visual, por ej. "He visto sombras, o figuras humanas o no-humanas cerca de mi cama, yo las he visto claramente y veo lo que hacen", táctil, por ej. "He tenido la experiencia de sentir una palmada en mi hombro, o cualquier otra sensación vívida de contacto físico de otra persona detrás mío, pero cuando me doy vuelta no veo a nadie"; e Hipnagógico / hipnopómpico, por ej. "Sólo cuando me estoy durmiendo o estoy despertando del sueño, he oído voces o diálogos, música o melodías, sonidos, a veces agradables y otras no, pero que escucho nítidamente").

Escala de Experiencias Disociativas (DES) (Bernstein y Putman, 1993; alfa de Cronbach $=.91$ ) una escala tri-factorial de 28 ítems en una escala Likert $0-10$ que mide una variedad de tendencias disociativas: amnesia, despersonalización y desrealización (por ej. "Hay 
personas que tienen la experiencia de conducir o estar viajando en un automóvil, colectivo o microbús y de repente se dan cuenta de que no recuerdan lo que pasó durante todo o parte del viaje").

Cuestionario de Experiencias Creativas (CEQ) (Merckelbach, Horselenberg y Muris, 2001; alfa de Cronbach $=.89$,) una escala de 25 ítems de respuesta verdadero/falso que mide la propensidad a la fantasía, especialmente la tendencia a fantasear en la niñez (por ej., "Cuando veo escenas de violencia por televisión, me siento tan involucrado en éstas que me provoca mucha inquietud").

Escala de Absorción de Tellegen (TAS) (Tellegen y Atkinson, 1974; alfa de Cronbach $=.91$ ) que mide en 34 ítems de respuesta verdadero/falso la frecuencia con que una persona se involucra en actividades que exigen atención y concentración (por ej. "Cuando escucho música de órgano u otra música imponente a veces siento como si me estuvieran levantando en el aire").

Se empleó también una medida de rasgos de personalidad el Cuestionario de Personalidad Esquizotípica (SPQ) (Raine, 1991; Raine, 1992, Raine y Baker, 1992; Raine y Benishay, 1995; alfa de Cronbach $=.91$ ) una escala tri-factorial de 74 ítems de respuesta dicotómica sí / no que mide estilos de pensamiento esquizotípicos (se empleó la puntuación total del SPQ y el subfactor 1 Cognitivo perceptual, por ej. “¿Alguna vez ha visto cosas que para los demás son invisibles?" o “ ¿Son sus pensamientos a veces tan fuertes que usted casi podría escucharlos?").

\section{Procedimiento}

Aplicamos una técnica de muestreo no-probabilística para obtener el mayor número de casos para analizar. El set de tests autoadministrables fue entregado en un sobre A4 a cada estudiante, en forma contrabalanceada, durante una clase. El tiempo promedio para completar los cuestionarios fue de 50 minutos (30 minutos para el grupo de pacientes). Los estudiantes recibieron una vaga información del objeto de estudio y se los invitó a participar voluntaria y anónimamente completando los test, en una única sesión, en días y horarios previamente pactados con los docentes. Ningún estudiante recibió compensación económica.

El orden de administración de ambas pruebas a los pacientes fue contrabalanceado y los cuestionarios de alucinaciones se presentaron bajo el pseudo-título de Cuestionario de Experiencias Psicológicas, con lo cual se evitó sesgar las respuestas. Los pacientes recibieron una vaga información del objeto de estudio y se les invitó a participar voluntaria y anónimamente en una única sesión, y tampoco recibieron compensación económica.

Los análisis fueron procesados mediante el SPSS 11.5 (en español) y los análisis estadísticos fueron evaluados a dos colas. A causa de la alta asimetría en los puntajes de las escala de alucinación, en lugar de un test t, aplicamos una $U$ de Mann-Whitney. 


\section{RESULTADOS}

Tabla N. ${ }^{0}$ 1. Porcentaje de encuestados en varias categorías demográficas.

\begin{tabular}{|c|c|c|}
\hline & $\begin{array}{c}\text { Grupo } \\
\text { no-clínico }\end{array}$ & $\begin{array}{l}\text { Grupo } \\
\text { clínico }\end{array}$ \\
\hline \multicolumn{3}{|l|}{ Estado Civil } \\
\hline Soltero & $208(97,2)$ & $24(80,0)$ \\
\hline Casado & $6(2,8)$ & 620,0 \\
\hline Separado / divorciado & - & - \\
\hline \multicolumn{3}{|l|}{ Orden de nacimiento } \\
\hline Hijo único & $15(7,0)$ & $7(23,3)$ \\
\hline Primer nacimiento & $74(34,6)$ & $9(30,0)$ \\
\hline Segundo nacimiento & $59(27,6)$ & $7(23,3)$ \\
\hline Ultimo hermano & $66(30,8)$ & $7(23,3)$ \\
\hline \multicolumn{3}{|l|}{ Religión } \\
\hline Católico & $171(79,9)$ & $26(86,7)$ \\
\hline Judío & $1(, 5)$ & - \\
\hline Cristiano no-denominacional & $9(4,2)$ & $1(3,3)$ \\
\hline No-denominacional & $12(5,6)$ & $1(10,3)$ \\
\hline Filosofías orientales & $3(1,4)$ & - \\
\hline Ateos & $18(8,4)$ & - \\
\hline \multicolumn{3}{|l|}{ Pensamiento político } \\
\hline Muy conservador & $3(1,4)$ & $1(3,3)$ \\
\hline Conservador & $24(11,2)$ & $15(50,0)$ \\
\hline Moderado & $117(54,7)$ & $11(36,7)$ \\
\hline Liberal & $65(30,4)$ & $3(10,0)$ \\
\hline Muy liberal & $5(2,3)$ & $1(3,3)$ \\
\hline \multicolumn{3}{|l|}{ Espiritualidad } \\
\hline No soy espiritual & $11(5,1)$ & $2(6,7)$ \\
\hline Soy un poco espiritual & $71(33,2)$ & $6(20,0)$ \\
\hline Soy moderadamente espiritual & $97(45,3)$ & $14(46,7)$ \\
\hline Soy muy espiritual & $31(14,5)$ & $7(23,3)$ \\
\hline Soy extremadamente espiritual & $3(1,4)$ & $1(3,3)$ \\
\hline
\end{tabular}

En base a las respuestas obtenidas de los estudiantes se conformaron dos grupos. A causa de la baja frecuencia, para agrupar a quienes tenían experiencias alucinatorias, se convirtieron las respuestas 1 (rara vez), 2 (ocasionalmente), 3 (a menudo) y 4 (muy frecuentemente) en un valor (Con experiencias $=1$ ). Para agrupar a quienes no tuvieron experiencias alucinatorias (Sin experiencias $=0$ ) se empleó la respuesta "Nunca". 
Predisposición hacia las eXPeriencias alucinatorias en Perú: eXaminando la continuidad noRmalidad-Patología

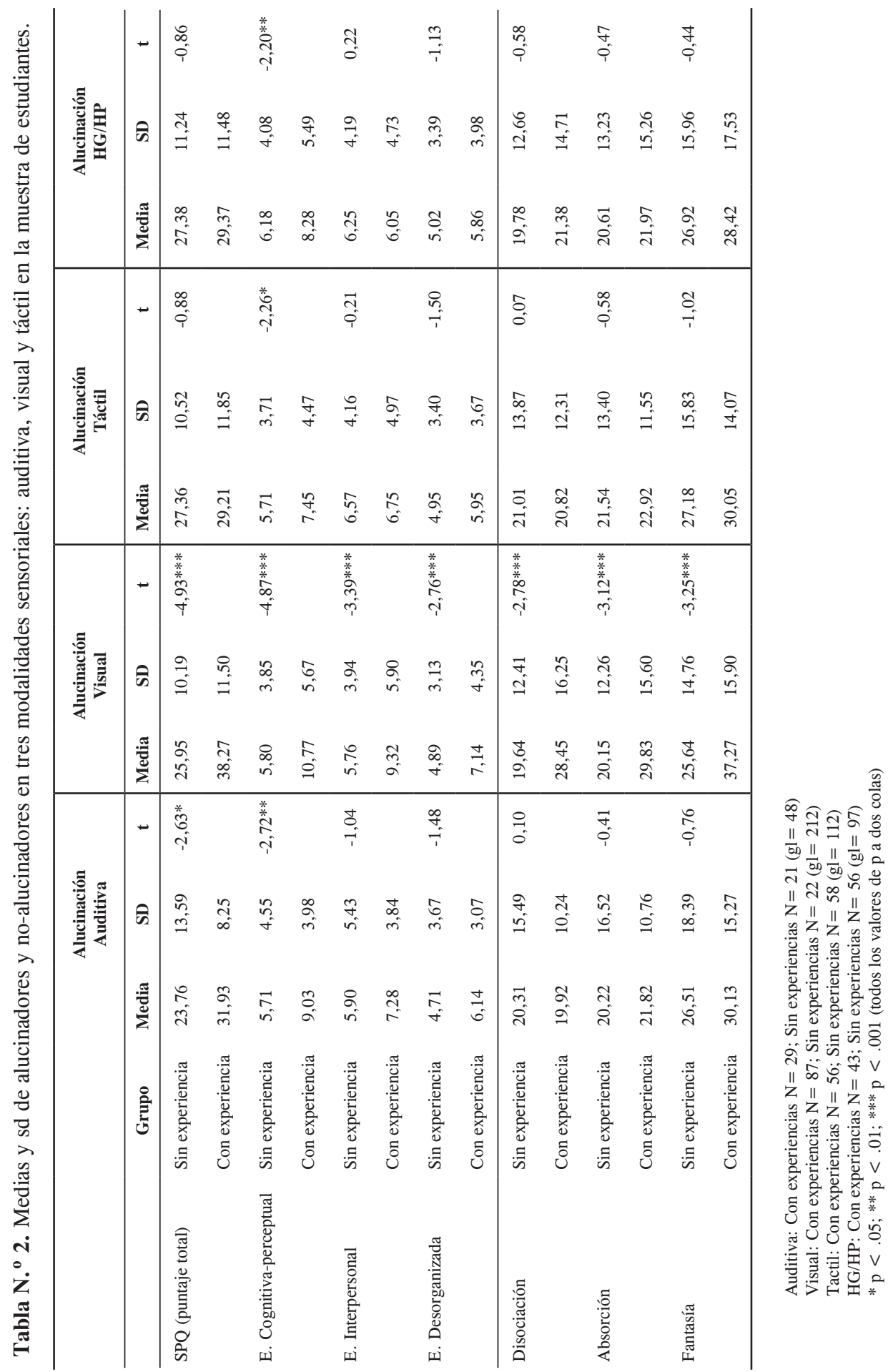


Propensión de esquizotipia. La Hipótesis 1 era que los estudiantes con experiencias alucinatorias puntuarían más alto que quienes no tuvieron experiencias en propensión a la esquizotipia (medido con el SPQ-A), lo cual se confirmó, en particular con el factor Cognitivo-perceptual (Auditiva $\mathrm{z}=2,72$; Visual: $\mathrm{z}=5.80 ; \mathrm{p}<.001$, a dos colas; y Táctil $\mathrm{z}=2.26$ y HG/HP z $=2.20, \mathrm{p}<.01$, a dos colas), donde la puntuación de esquizotipia para quienes tuvieron la experiencia alucinatoria visual era significativamente más alta que quienes no tuvieron la experiencia (ver Tabla N. ${ }^{\circ} 2$ ). Respecto a los factores interpersonal y desorganizada, también se confirmó sólo para la modalidad visual $(\mathrm{z}=<.001$ y .007 , a dos colas, respectivamente)

Disociación: La Hipótesis 2 era que los estudiantes con experiencias alucinatorias puntuarían más alto que quienes no tuvieron experiencias en Experiencias disociativas (medido con el DES-2), lo cual se confirmó para la modalidad Visual $(\mathrm{z}=2.78 ; \mathrm{p}<.006$, a dos colas) donde la puntuación de disociación para quienes tuvieron la experiencia era significativamente más alta que quienes no tuvieron la experiencia (ver Tabla N. ${ }^{\circ} 2$ ).

Absorción: La Hipótesis 3 era que los estudiantes con experiencias alucinatorias puntuarían más alto que quienes no tuvieron experiencias en el puntaje de absorción psicológica (medido con el TAS), lo cual también se confirmó para la modalidad Visual $(\mathrm{z}=3.12$; $\mathrm{p}<$ .002 , a dos colas) donde la puntuación de disociación para quienes tuvieron la experiencia alucinatoria visual era significativamente más alta que quienes no tuvieron la experiencia (ver Tabla N. ${ }^{\circ}$ 2). No se confirmó para ninguna de las otras modalidades de alucinación.

Tabla N. ${ }^{\circ}$ 3. Comparación entre los grupos clínico y no-clínico (estudiantes).

\begin{tabular}{|c|c|c|c|c|c|}
\hline Variable & Grupo $^{(a)}$ & Media & SD & $\mathbf{U}$ & $\mathbf{z}$ \\
\hline \multirow[t]{2}{*}{ Alucinación auditiva } & Clínico & 23,63 & 15,80 & 953,0 & $6,24 * * *$ \\
\hline & No-clínico & 6,46 & 5,37 & & \\
\hline \multirow[t]{2}{*}{ Alucinación visual } & Clínico & 10,47 & 8,41 & 1065,0 & $6,10 * * *$ \\
\hline & No-clínico & 1,99 & 2,66 & & \\
\hline \multirow[t]{2}{*}{ Alucinación táctil } & Clínico & 6,03 & 4,31 & 1154,0 & $5,77 * * *$ \\
\hline & No-clínico & 2,00 & 2,15 & & \\
\hline \multirow[t]{2}{*}{ Alucinación HG/HP } & Clínico & 9,00 & 5,57 & 856,50 & $6,58 * * *$ \\
\hline & No-clínico & 2,43 & 2,64 & & \\
\hline \multirow[t]{2}{*}{ Esquizotipia (Total) } & Clínico & 27,27 & 9,08 & 2763,0 & n.s. \\
\hline & No-clínico & 30,92 & 11,51 & & \\
\hline \multirow[t]{2}{*}{ E. Cognitiva-perceptual } & Clínico & 13,90 & 4,97 & 1319,0 & $5,23 * * *$ \\
\hline & No-clínico & 8,46 & 4,87 & & \\
\hline \multirow[t]{2}{*}{ E. Interpersonal } & Clínico & 7,37 & 2,47 & 2704,50 & n.s. \\
\hline & No-clínico & 7,11 & 4,73 & & \\
\hline \multirow[t]{2}{*}{ E. Desorganizada } & Clínico & 4,97 & 1,86 & 2903,0 & n.s. \\
\hline & No-clínico & 5,97 & 3,75 & & \\
\hline \multirow[t]{2}{*}{ Disociación } & Clínico & 4,37 & 1,76 & 28130 & n.s. \\
\hline & No-clínico & 5,91 & 3,75 & & \\
\hline \multirow[t]{2}{*}{ Absorción } & Clínico & 43,32 & 20,9 & 1694,50 & $4,18 * * *$ \\
\hline & No-clínico & 24,88 & 14,0 & & \\
\hline \multirow[t]{2}{*}{ Fantasía } & Clínico & 43,96 & 18,0 & 2094,50 & 3,08 *** \\
\hline & No-clínico & 26,01 & 14,3 & & \\
\hline
\end{tabular}

(a) Grupo clínico $\mathrm{N}=30$; grupo no-clínico $\mathrm{N}=214$

** $\mathrm{p}=0,01 ; \mathrm{p}=0,05$ (bilateral). 
En la Tabla N. ${ }^{\circ} 3$, presentamos los resultados de la comparación de puntajes entre ambas muestras (clínica y no-clínica). La muestra clínica reveló puntajes altos las cuatro modalidades de experiencias alucinatorias, esquizotipia cognitivo-perceptual (excepto interpersonal y desorganizada), disociación, absorción y propensidad a la fantasía (todas las $\mathrm{p}<.001$, a dos colas).

Tabla N. ${ }^{\circ}$ 4. Correlación Pearson entre experiencias alucinatorias, propensidad a la esquizotipia, disociación, absorción y fantasía en la muestra clínica (pacientes).

\begin{tabular}{lccccccccccc}
\hline \multicolumn{1}{c}{ Variable } & $\mathbf{1}$ & $\mathbf{2}$ & $\mathbf{3}$ & $\mathbf{4}$ & $\mathbf{5}$ & $\mathbf{6}$ & $\mathbf{7}$ & $\mathbf{8}$ & $\mathbf{9}$ & $\mathbf{1 0}$ & $\mathbf{1 1}$ \\
\hline 1. Alucinación auditiva & -- &, 593 &, 513 &, 677 &, 314 &, 477 &, 136 &, 200 &, 521 &, 532 &, 531 \\
2. Alucinación visual & & -- &, 698 &, 692 &, 266 &, 513 &, 106 &, 140 &, 423 &, 427 &, 452 \\
3. Alucinación táctil & & & -- &, 745 &, 265 &, 495 &, 103 &, 178 &, 370 &, 375 &, 385 \\
4. Alucinación HG/HP & & & & -- &, 233 &, 429 &, 113 &, 171 &, 439 &, 444 &, 464 \\
\hline 5. Esquizotipia (Total) & & & & -- &, 704 &, 681 &, 689 &, 478 &, 457 &, 440 \\
6. E. cognitiva-perceptual & & & & & -- &, 287 &, 537 &, 537 &, 531 &, 532 \\
7. E. interpersonal & & & & & & & -- &, 566 &, 309 &, 299 &, 267 \\
8. E. desorganizada & & & & & & & & -- &, 364 &, 380 &, 331 \\
\hline 9. Disociación & & & & & & & & -- &, 886 &, 835 \\
10. Absorción & & & & & & & & & -- &, 859 \\
11. Fantasía & & & & & & & & & & - \\
\hline
\end{tabular}

La Tabla N. ${ }^{\circ} 5$ presenta las correlaciones entre diferentes medidas de experiencias perceptuales anómalas. De las 55 correlaciones Pearson, 48 (87\%) fueron significativas en la muestra de pacientes. Los valores en negrita muestran aquellas correlaciones cuya significación están a un nivel de $\mathrm{p}<.001$ ( $\mathrm{p}$ ajustada a 55 casos a un nivel de .05). Estas correlaciones también muestran que aquellos que tuvieron experiencias alucinatorias tuvieron una tendencia (comparados con aquellos que no tuvieron la experiencia) a tener otra experiencia. Pero debemos reconocer que estas correlaciones no son relaciones de causa y efecto.

Tabla N. ${ }^{0}$ 5. Correlación Pearson entre experiencias alucinatorias, propensidad a la esquizotipia, disociación, absorción y fantasía en la muestra no-clínica (estudiantes).

\begin{tabular}{|c|c|c|c|c|c|c|c|c|c|c|c|}
\hline Variable & 1 & 2 & 3 & 4 & 5 & 6 & 7 & 8 & 9 & 10 & 11 \\
\hline 1. Alucinación auditiva & -- & ,919 &, 830 &, 873 & , 468 & ,435 & , 460 & , 481 & 846 & 848 & ,738 \\
\hline 2. Alucinación visual & & -- &, 870 & ,876 & ,439 & ,431 & ,423 & , 449 &, 728 & ,742 & ,602 \\
\hline 3. Alucinación táctil & & & -- & ,922 &, 315 & ,303 & ,329 & ,300 & ,612 & 607 &, 503 \\
\hline 4. Alucinación HG/HP & & & & -- & , 418 & ,397 & ,446 & ,345 & ,635 & ,636 & ,491 \\
\hline 5. Esquizotipia (Total) & & & & & -- & ,967 & ,974 & ,924 & , 405 &, 519 & ,391 \\
\hline 6. E. Cognitiva-perceptual & & & & & & -- & 899 &, 850 & ,397 & ,494 & ,369 \\
\hline 7. E. Interpersonal & & & & & & & -- & ,886 &, 370 & ,490 & 357 \\
\hline 8. E. Desorganizada & & & & & & & & -- & ,434 &, 550 & ,449 \\
\hline 9. Disociación & & & & & & & & & -- & ,973 & ,937 \\
\hline 10. Absorción & & & & & & & & & & -- & ,922 \\
\hline 11. Fantasía & & & & & & & & & & & -- \\
\hline
\end{tabular}


La Tabla N. ${ }^{\circ} 5$ presenta correlaciones entre las diferentes experiencias de los estudiantes. De 55 correlaciones Pearson, 31 (56\%) fueron significativas. Los valores en negrita muestran correlaciones significativas en un nivel de $\mathrm{p}<.001$ (p ajustada a 33 casos a un nivel de .05). Estas correlaciones muestran que aquellos participantes que tuvieron una de las experiencias alucinatorias tuvieron la tendencia (comparados con quienes no tuvieron la experiencia) de tener otra experiencia.

\section{DISCUSIÓN}

Estos resultados sugieren que los estudiantes con experiencias perceptuales anómalas indicaron algún grado de propensión a la esquizotipia perceptual, que no parece estar relacionada con las habilidades sociales e interpersonales. De igual modo, individuos saludables con alta tendencia a la disociación, absorción y propensidad a la fantasía mostraron algún indicador de alucinación visual, lo cual parece confirmar una de las dos dimensiones de esquizotipia (esquizotipia positiva), en donde individuos que puntúan alto en esta escala (experiencias perceptuales no convencionales) experimentan un funcionamiento que incluye varias formas de alucinaciones, ideación paranoide, ideas de referencia y trastornos del pensamiento, que a menudo carecen de consecuencias patológicas negativas que se encuentran, por el contrario, en aquellos individuos afectados por dificultades para experimentar placer a nivel físico y social, y aplanamiento afectivo. En efecto, otros resultados confirmaron diferencias significativas entre ambas muestras (clínica y no-clínica), en experiencias alucinatorias, esquizotipia cognitivo-perceptual, disociación, absorción y propensidad a la fantasía.

Se puede concluir que este estudio muestra que muchas más personas experimentan alucinaciones que las que entran en contacto con los servicios médicos o psiquiátricos. Otros estudios similares han explorado la hipótesis del continuo de las experiencias psicóticas en población saludable, pero, por el contrario, han reagrupado la muestra en puntajes altos vs. puntajes bajos en un intento por determinar la potencia de la diferencia. Con todo, sus resultados no muestran sustanciales diferencias con nuestro estudio, en comparación con la población saludable. El principal problema de estos estudios es que se incluye en la misma población al subgrupo de puntajes "alto" de experiencias alucinatorias, que debería ser considerado como "individuos no hospitalizados", que forman parte de la sociedad en general, pero no representan a individuos saludables (quienes puntúan bajo). Además muchos de estos estudios limitan el tamaño de la muestra a unos pocos individuos, en lugar de examinar el continuo de experiencias que se distribuye normalmente. Nuestro análisis, en consecuencia, es más sensible y relevante que muestras seleccionadas por puntajes, y permite explorar la hipótesis de continuo más eficazmente.

La frecuencia de la experiencia alucinatoria es un valor que debe ser cuidadosamente considerado por varias razones. Primero, puede ser resultado de deseabilidad social, una aversión de hablar sobre cuestiones íntimas, que induce a ciertos sujetos a guardar silencio sobre síntomas considerados vergonzantes o que puede indicar que tienen severos trastornos mentales. El anonimato que se garantiza mediante una encuesta mitiga estos sesgos, y se sientan menos avergonzados a responder, y quizá ser más sinceros que una entrevista cara a cara. Segundo, muchos individuos con alucinaciones no advierten sus problemas y pueden reportar confusamente tales experiencias. 
Las alucinaciones hipnagógicas-hipnopómpicas necesitan ser diferenciadas de otros tipos de alucinaciones en el sentido de que ocurren solamente en el período transicional del despertar al dormir (hipnagógicas) o del dormir al despertar (hipnopómpicas). La mayoría de los individuos que experimentaron estas alucinaciones saben que lo que perciben no es verdadero. Si un individuo se aterroriza por lo que percibe, quizás intente escapar de las alucinaciones y varias veces injuria a otros o a sí mismo. Hay casos en la literatura de narcolepsia en donde este tipo de experiencia ha sido incorrectamente diagnosticada como trastorno psicopático (Douglas, 1994; Sartorius, Shapiro y Jablensky, 1974).

La presencia de alucinación no es equivalente de esquizofrenia. Las condiciones de vida pueden generar este fenómeno. Las alucinaciones son síntomas de trastorno psicótico sólo cuando el individuo cree firmemente en éstas como verdaderas, menospreciando lo que la gente dice a su alrededor.

Finalmente, utilizar a la población no hospitalizada nos permite determinar cuántos individuos con síntomas de psicosis y alucinaciones están viviendo en la sociedad y tienen, al menos, una función social. En síntesis, varias fuentes independientes indican que la psicosis existe como un continuo de experiencias, con una distribución en la población general. Sus implicaciones para el diagnóstico (Jones, Cormac, Mota, y Campbell, 1999), etiología y tratamiento de los estados psicóticos asociados con la necesidad de una atención especializada van claramente en aumento, y se puede recomendar la incorporación de una noción de continuo en la psicosis en la investigación científica y la práctica clínica.

\section{NOTA DE RECONOCIMIENTO}

Este estudio fue posible gracias al financiamiento de la Fundación BIAL de Portugal. Agradecemos también al Dr. William Acuña de la Facultad de Psicología de la Universidad Ricardo Palma de Lima y al Lic. Pedro Tapia por su cooperación para la administración de los cuestionarios.

\section{REFERENCIAS BIBLIOGRÁFICAS}

1. Aleman. A. y de Haan, E.H.F. (1998). On redefining hallucination. American Journal of Orthopsychiatry, 68, 656-658.

2. Barrett, T., y Etheridge, J. B. (1992). Verbal hallucinations in normals, I: People who hear "voices." Applied Cognitive Psychology, 6, 379-387.

3. Barrett, T. R.. y Etheridge, J. B. (1994). Verbal hallucinations in normals. Part 3: Dysfunctional personality correlates. Personality and Individual Differences, 16, 57-62.

4. Bentall, R. P. (2000). Hallucination experiences. En E. Cardeña, S.J. Lynn, y S. Krippner (eds.), Varieties of anomalous experiences: Examining the scientific evidence (pp.85-120). Washington, DC: American Psychological Association.

5. Bentall, R. P., y Slade, P. D. (1985a). Reliability of a measure of disposition towards hallucinations. Personality and Individual Differences, 6, 527-529. 
6. Bentall, R. P., y Slade, P. D. (1985b). Reality testing and auditory hallucinations: A signal detection analysis. British Journal of Clinical Psychology, 3, 159-169.

7. Bernstein, E. M., y Putnam, F. W. (1986). Development, reliability and validity of a dissociation scale. Journal of Nervous and Mental Disease, 174, 727-735.

8. Chapman, L.J., Edell, E.W, y Chapman, J.P. (1980). Physical anhedonia, perceptual aberration and psychosis proneness. Schizophrenia Bulletin, 6, 639-653.

9. Chapman, L.J., Chapman, J.P., Kwapil, T.R., Eckblad, M., y Zinser, M.C. (1994) Putatively psychosis-prone subjects 10 years later. Journal of Abnormal Psychology, 103, 171-183.

10. Claridge, G. (1997a). Schizotypy: Implications for illness and health. Oxford: Oxford University Press.

11. Claridge, G. (1997b). Theoretical background and issues. En G. Claridge (Ed.), Schizotypy: Implications for illness and health (pp. 3-19). Oxford: Oxford University Press.

12. Douglas, N.J. (1994), Breathing during sleep in normal subjects. En R. Cooper (Ed.), Sleep (pp. 76-95). Chapman: London.

13. Johns, L.C. y van Os, J. (2001) The continuity of psychotic experiences in the general population. Clinical Psychology Review, 21, 1125-1141.

14. Jones, C., Cormac, I., Mota, J. y Campbell, C. (1999). Cognitive behaviour therapy for schizophrenia. En The Cochrane Library. Oxford, England: Update Software.

15. Kwapil, T.R., Miller, M.B., Zinser, M.C., Chapman, J. y Chapman, L.J. (1997) Magical ideation and social anhedonia as predictors of psychosis proneness: A partial replication. Journal of Abnormal Psychology, 106, 491-495.

16. Leudar, I., Thomas, P., McNally, D. y Glinsky, A. (1997). What can voices do with words? Pragmatics of verbal hallucinations. Psychological Medicine, 27, 885-898.

17. Merckelbach, H; Horselenberg, R. y Muris, P. (2001). The Creative Experiences Questionnaire (CEQ): A brief self report measure of fantasy proneness. Personality and Individual Differences, 31, 987995.

18. Ohayon, M. M., Priest, R. G., Caulet, M., \& Guilleminault, C. (1996). Hypnagogic and hypnopompic hallucinations: Pathological phenomena? British Journal of Psychiatry, 169, 459-467.

19. Posey, T. B., y Losch, M. E. (1983). Auditory hallucinations of hearing voices in 375 normal subjects. Imagination, Cognition and Personality, 2, 99-113.

20. Raine, A. (1991). The SPQ: A scale for the assessment of schizotypy personality based on DSM-III-R criteria. Schizophrenia Bulletin, 17, 555-564.

21. Raine, A. (1992). Sex differences in schizotypal personality in a non-clinical population. Journal of Abnormal Psychology, 101, 361-364. 
22. Raine, A. y Baker, L. (1992) The Schizotypal Personality Questionnaire: Genetics, Psychophysiology. Neuropsychology and Gender Differences. Western Psychological Association, Portland, Oregon.

23. Raine, A. y Benishay, D. (1995). The SPQ-B: A brief screening instrument for schizotypal personality disorder. Journal of Personality Disorders, 9, 346-355.

24. Sartorius, N., Shapiro, R., y Jablensky, A. (1974). The international pilot study of schizophrenia. Schizophrenia Bulletin, 1, 21-25.

25. Sidgwick, H. A. (1894). Report of the census on hallucinations. Proceedings of the Society for Psychical Research, 26, 259-394.

26. Tellegen, A. (1982) The Multidimensional Personality Questionnaire. Minneapolis. University of Minnesota.

27. Tellegen, A., y Atkinson, G. (1974). Openness to absorbing and self-altering experiences ('absorption'), a trait related to hypnotic susceptibility. Journal of Abnormal Psychology, 83, 268-277.

28. Tien, A.Y. (1991). Distribution of hallucinations in the population. Social Psychiatry and Psychiatric Epidemiology, 26, 287-292.

29. Watkins, J. (2003). Oír voces: Una experiencia habitual. Buenos Aires: Deva's.

30. Wilson, S. C., y Barber, T. X. (1981). Vivid fantasy and hallucinatory abilities in the life histories of excellent hypnotic subjects ("somnambules"): Preliminary report with female subjects. En E. Klinger (Ed.), Imagery: Concepts, results, and applications, Vol. 2. (pp. 133-149). New York: Plenum.

31. Wilson, S. C. y Barber, T. X. (1983). The fantasy-prone personality: Implications for understanding imagery, hypnosis, and parapsychological phenomena. En A. A. Sheikh (Ed.), Imagery: Current theory, research, and application (pp. 340-390). New York: Wiley.

32. Young, H. F., Bentall, R. P., Slade, P. D., y Dewey, M. E. (1986). Disposition towards hallucinations, gender and IQ score. Personality and Individual Differences, 7, 247-249. 